\title{
UDOT Signal Performance Metrics: Configuration
}

\author{
Jamie Mackey \\ UDOT Statewide Signal Engineer \\ jamiemackey@utah.gov
}




\section{http://udottraffic.utah.gov/signalperformancemetrics}

\section{느엉 Signal Performance Metrics \\ AASHTC}

- Metric Type

Purdue Phase Termination

Split Monitor

Purdue Coordination Diagram

Approach Volume

Approach Delay

Arrivals on Red

Speed

Turning Movement Counts

Yellow and Red Actuations

Purdue Split Failure

Pedestrian Delay

Transitions

Preemption Details

Transit Signal Priority Details 


\section{Configuration Utility - Basic Metrics}

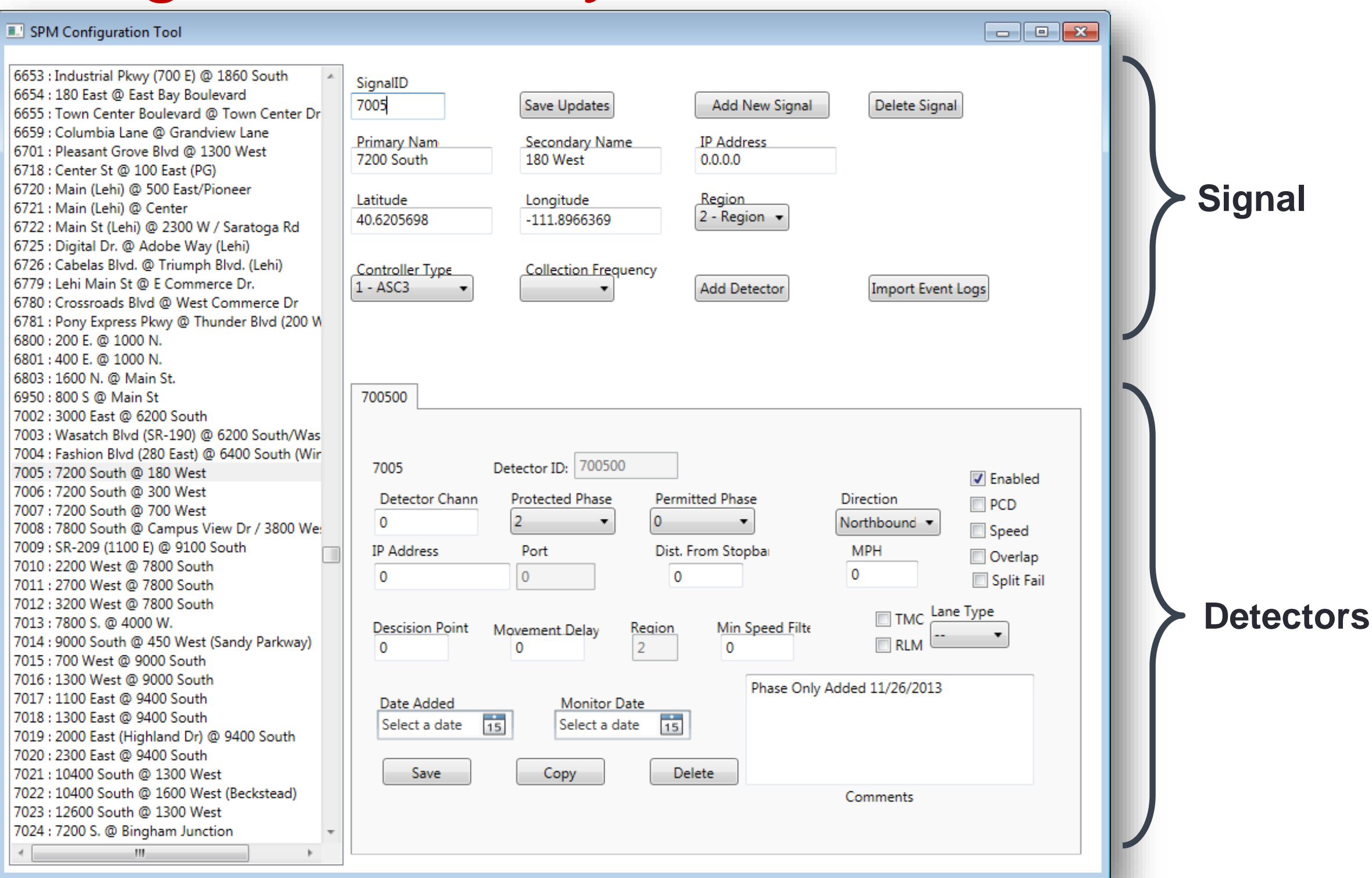




\section{Configuration Utility - Basic Metrics}

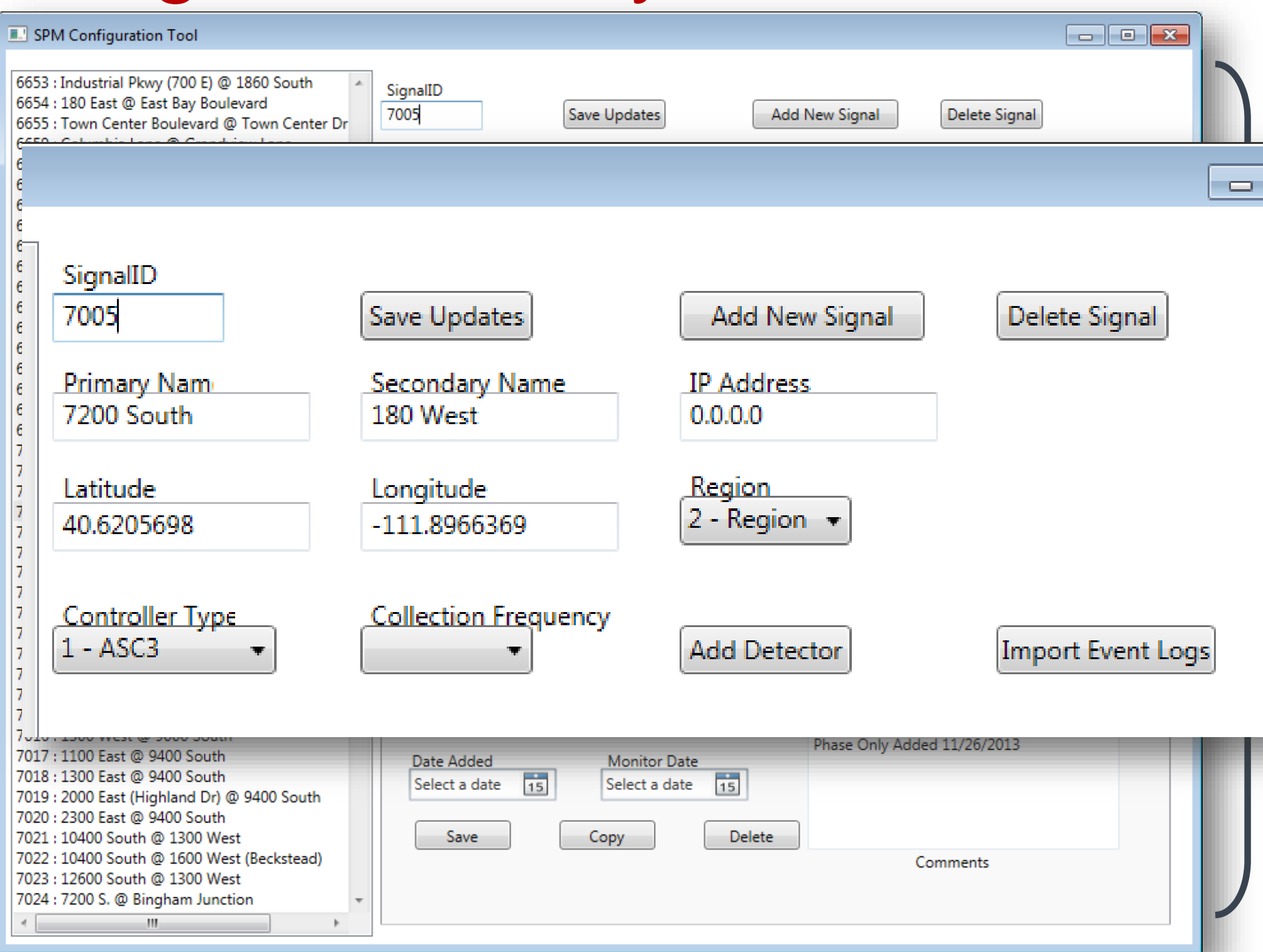




\section{Configuration Utility - Basic Metrics}

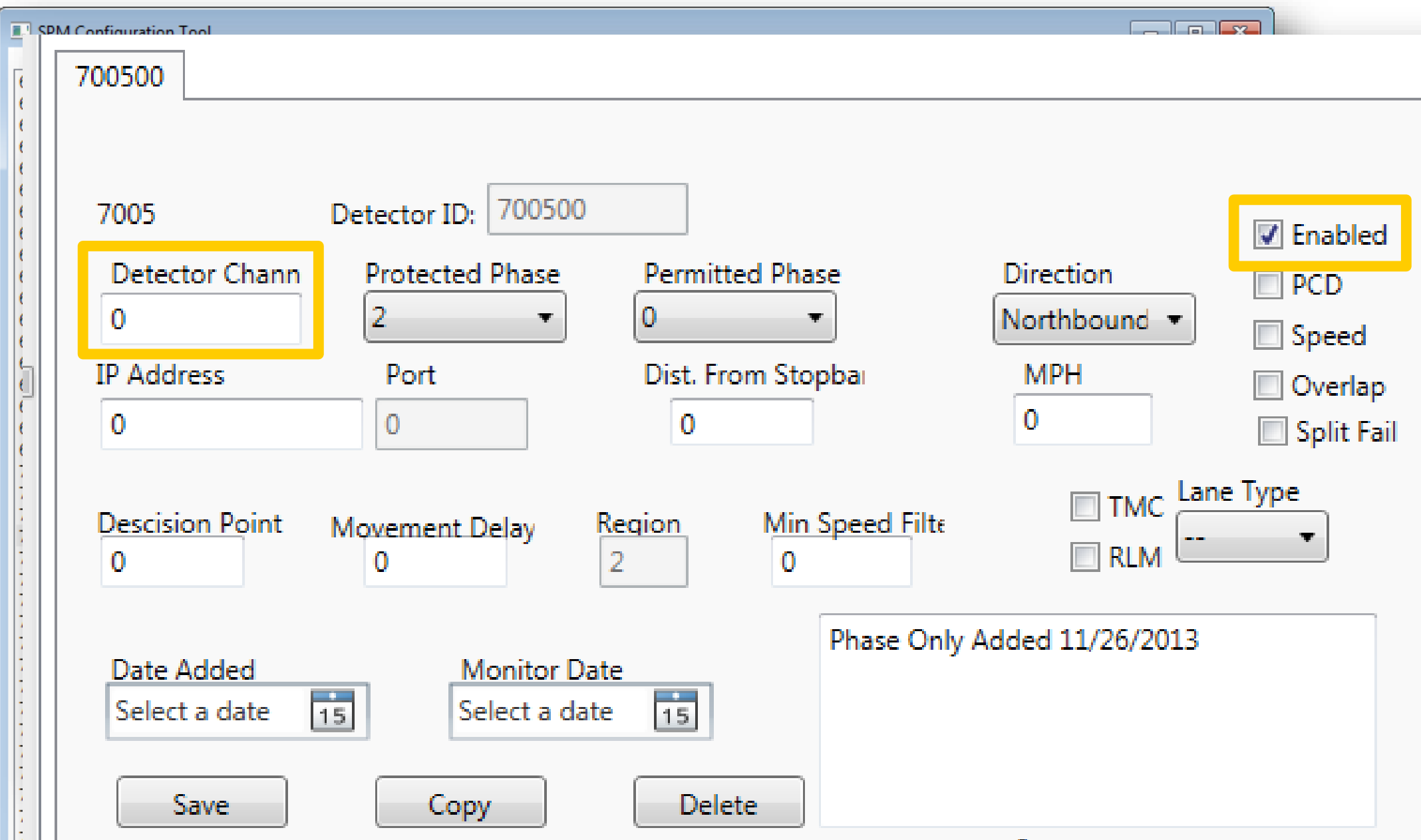

Comments 


\section{Configuration Utility - Purdue Coordination Configuration Utility - Diagram}

\begin{tabular}{|l|l|l|}
\hline 702300 & 702310 & 702312 \\
\hline
\end{tabular}
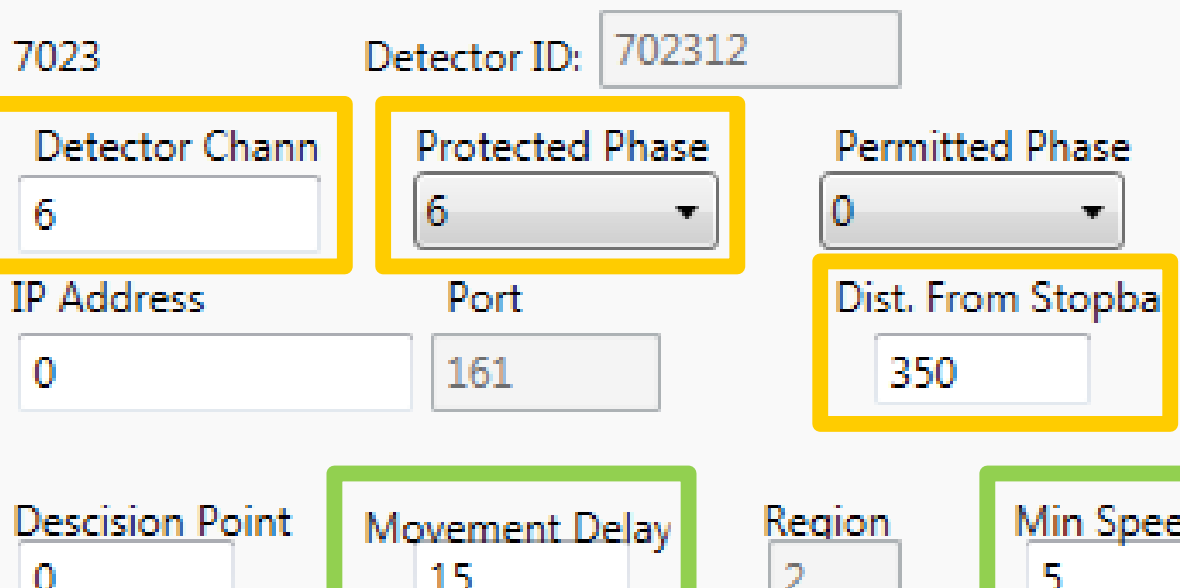

0
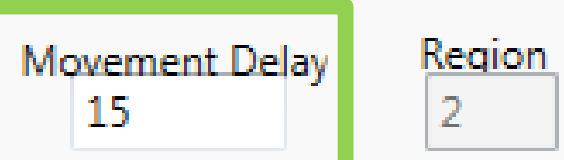
Min Speed Filte 5

$7 / 11 / 0214$
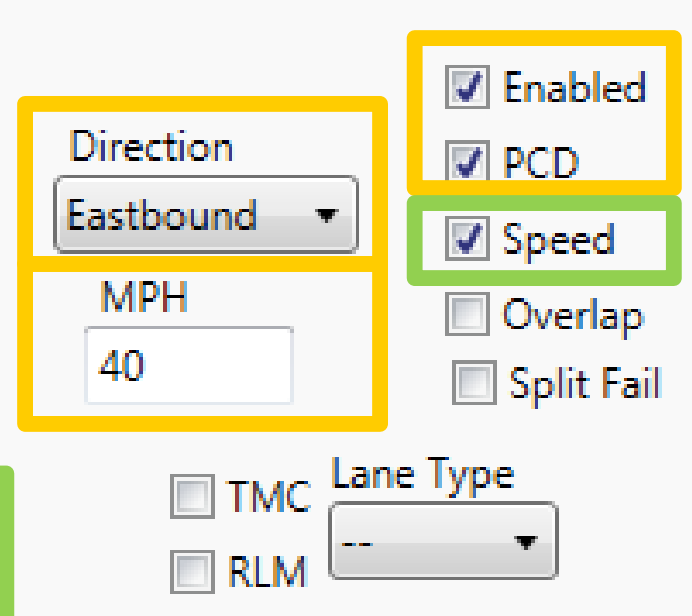

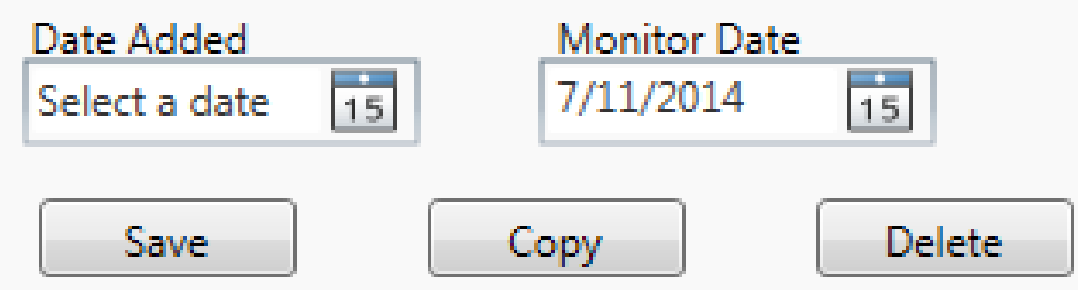




\section{Configuration Utility - Turning Movement Counts}

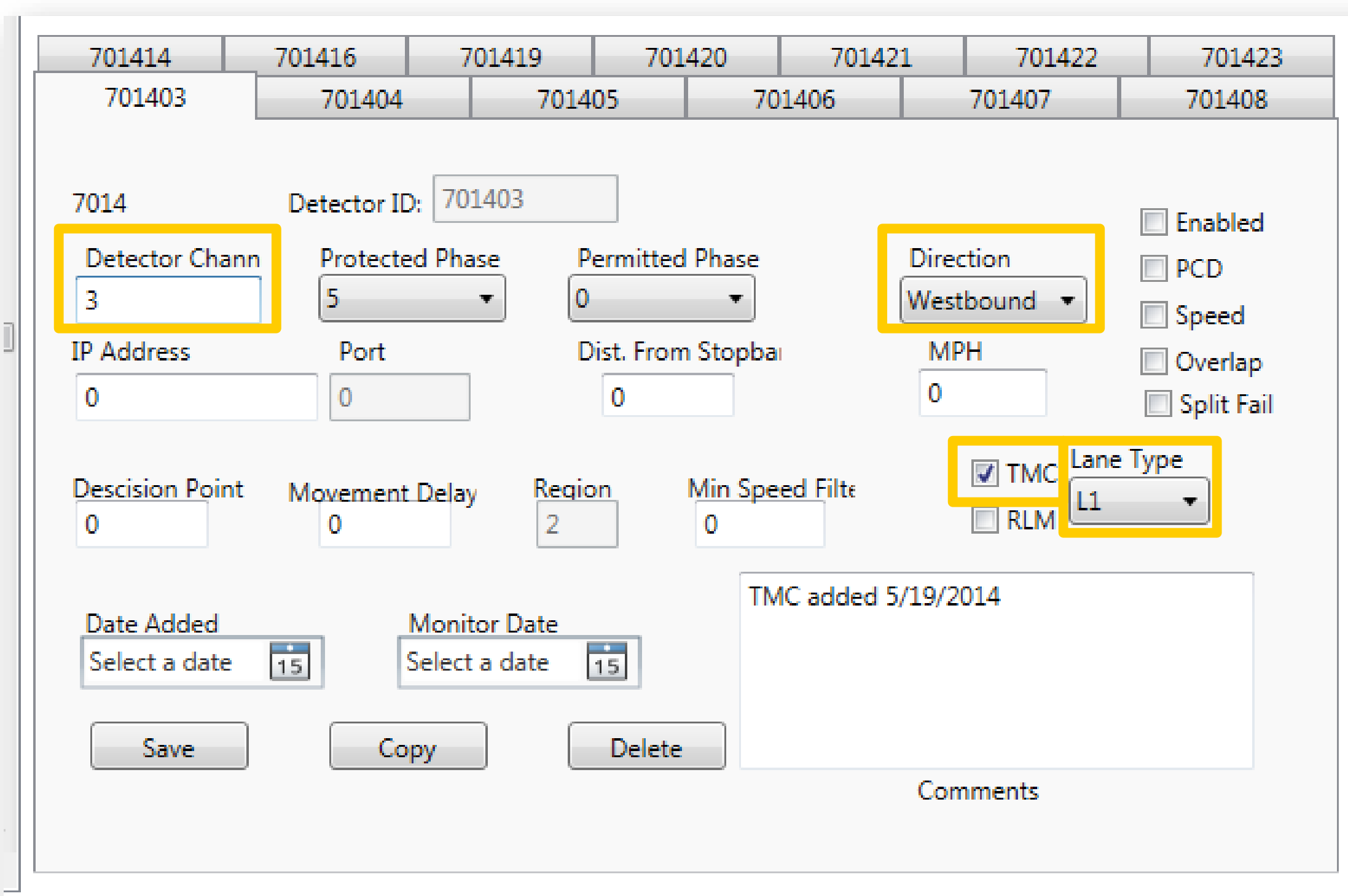




\section{Configuration Utility - Yellow \& Red Actuations}

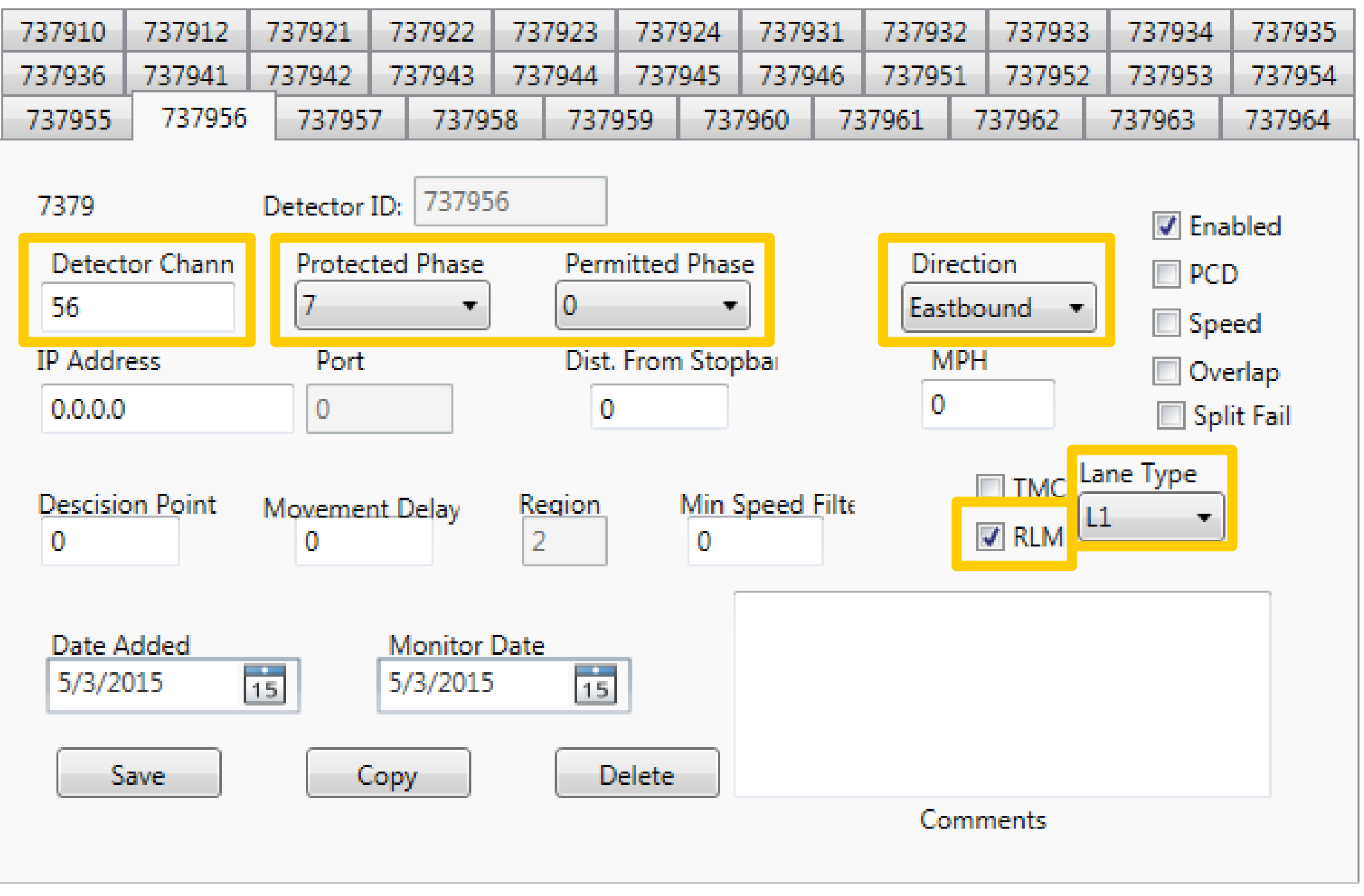




\section{Configuration Utility - Purdue Split Failure}

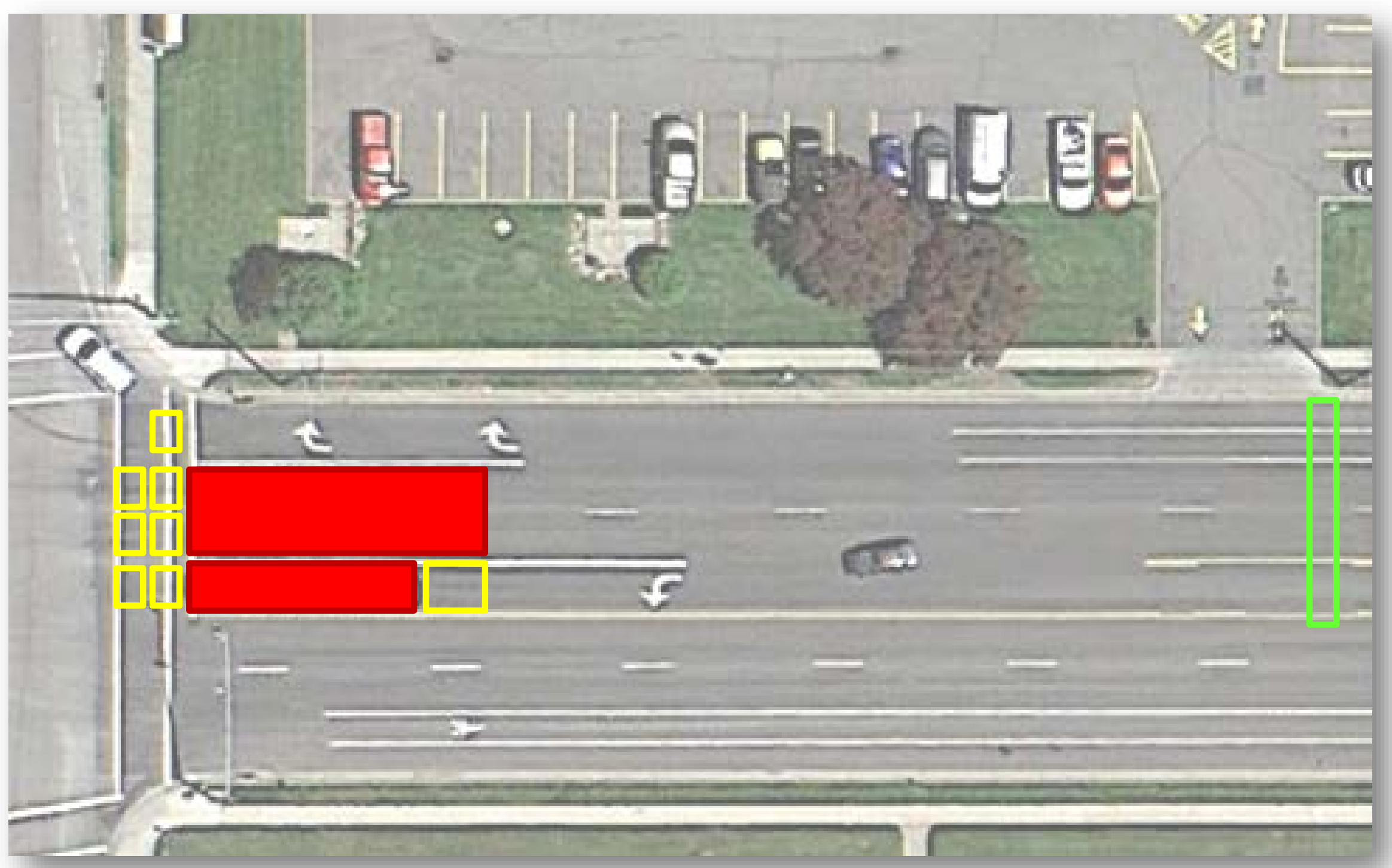




\section{Configuration Utility - Purdue Split Failure}

\begin{tabular}{|c|c|c|c|c|c|}
\hline 735512 & 735513 & 735514 & 735516 & \multicolumn{2}{|c|}{735517} \\
\hline 735523 & 735524 & 735525 & 735526 & 735527 & 73552 \\
\hline 735500 & 735502 & 735503 & 735504 & 735505 & 73550 \\
\hline 7355 & \multicolumn{2}{|r|}{ Detector ID: } & 735502 & & \\
\hline \multicolumn{2}{|c|}{ Detector Chann } & \multicolumn{2}{|c|}{ Protected Phase } & \multicolumn{2}{|c|}{ Permitted Phase } \\
\hline 2 & & & 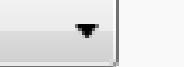 & 2 & $\cdot$ \\
\hline \multicolumn{2}{|c|}{ IP Address } & Port & & \multicolumn{2}{|c|}{ Dist. From Stopba } \\
\hline \multicolumn{2}{|l|}{0.0 .0 .0} & 0 & & \multicolumn{2}{|c|}{0} \\
\hline
\end{tabular}

$\begin{array}{lcccc}\text { Descision Point } & \begin{array}{c}\text { Movement Delay } \\ 0\end{array} & \text { Region } & \text { Min Speed Filte } & \square \text { TMC Lane Type } \\ 0 & 2 & 0 & \square \text { RLM L1 }\end{array}$

\begin{tabular}{|c|c|c|c|}
\hline \multicolumn{2}{|l|}{ Date Added } & \multicolumn{2}{|c|}{ Monitor Date } \\
\hline $11 / 24 / 2015$ & 15 & $11 / 24 / 2015$ & 10 \\
\hline Save & & Copy & Delete \\
\hline
\end{tabular}




\section{Database Access}

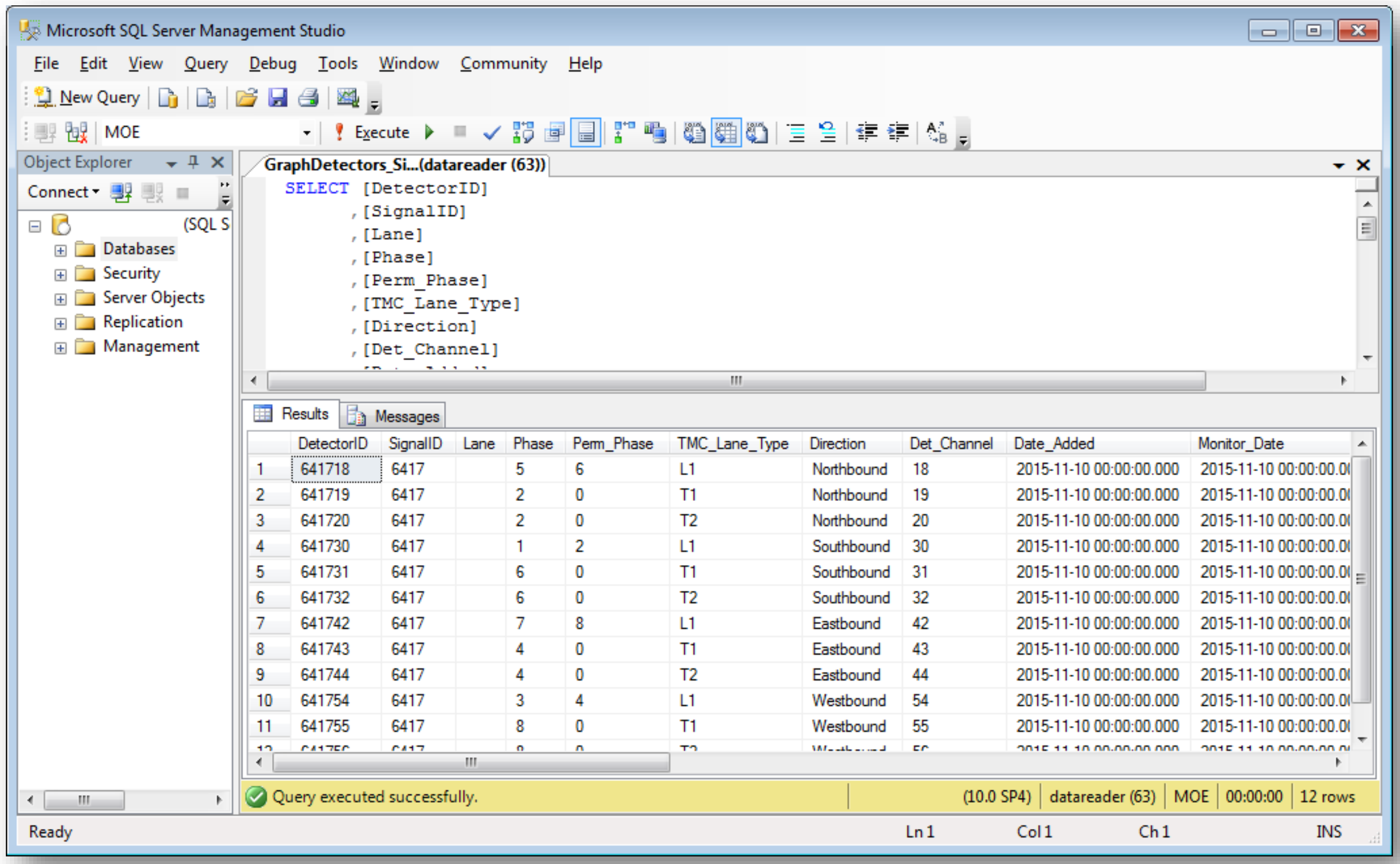




\section{UDOT Configuration Stats}

Detector Count by Metric

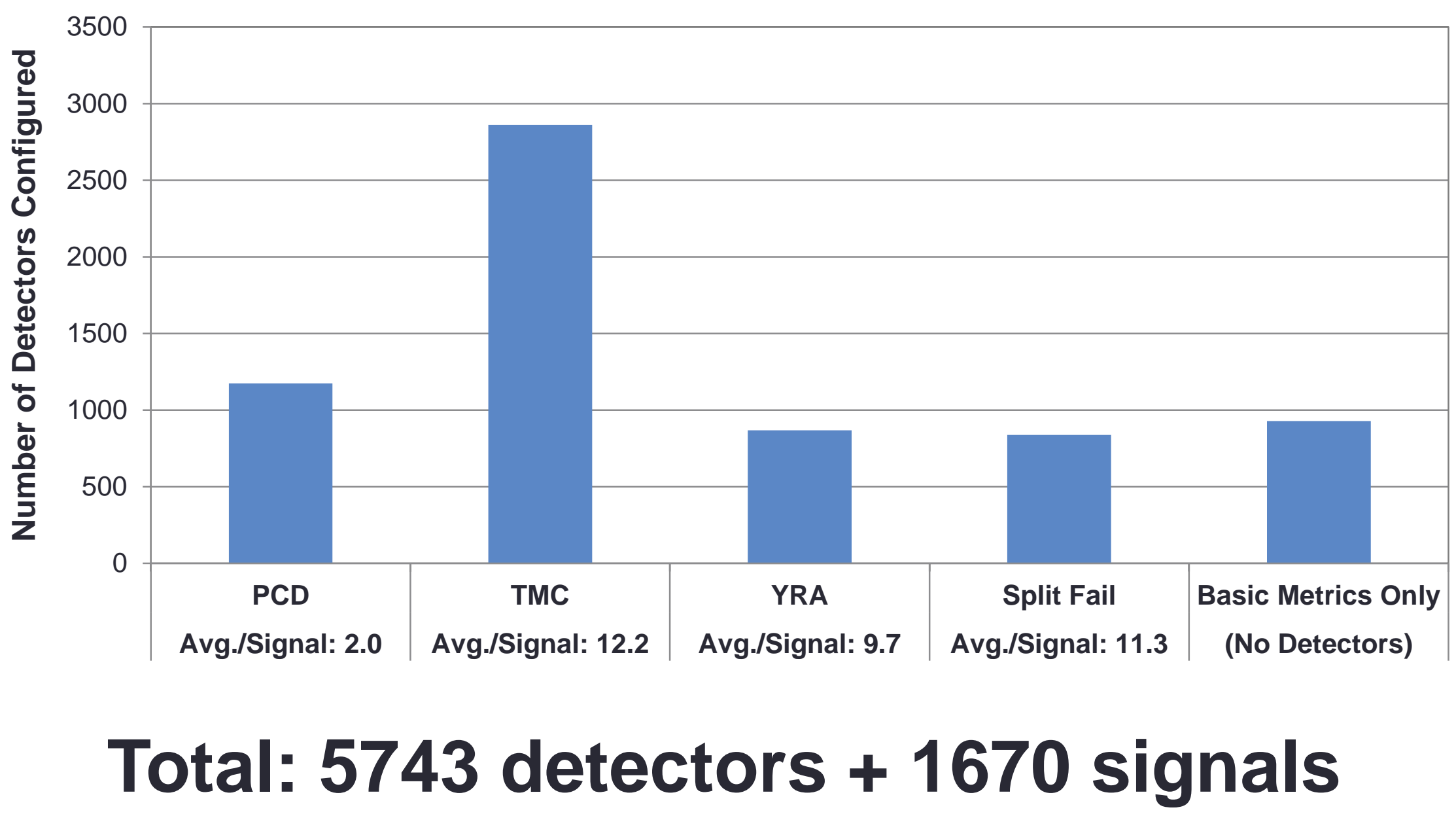




\section{Signal Numbering}

Utah Statewide Signal Numbering Convention

- Region 1

- UDOT

- Non-UDOT

- Region 2

- UDOT

$7000-7999$

- Salt Lake City

- Salt Lake County $4000-4999$

- Region 3

- UDOT

- Non-UDOT

- Region 4

- UDOT

- Non-UDOT

- Other

- Ramp Meters 8000 - 8099

- Test controllers $9000-9999$
$6000-6499$

$6500-6999$

$8100-8599$

$8600-8899$

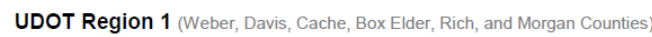

Master Database 


\section{Signal Numbering}

Utah Statewide Signal Numbering Convention

- Region 1
- UDOT
- Non-U[

- Region 2

- UDOT

- Salt Lal

- Salt Lal

- Region $ミ \bullet \bullet$

- UDOT •

- Non-U[ - *nertss

- Region $\triangle$ - UDOT

- Non-U[

- Other

- Ramp N. Sout no - owsonse

\section{Number: 7282}

Name: 3500 S (SR-171) @ 4000 W

Type: Econolite ASC/3 (Full) v2.61 (NTCIP) IP/Port:

Main Street: 3500 S (SR-171)

Side Street: 4000 W

Pattern: Pattern 7

Status: Coord

Active Alarms: No active alarms.

- Test controllers 9000 - 9999

UDOT Region 1 (Weber,
UDOT Signals: $5000-5499$

Box Elder, Rich, and Morgan Counties)

Non-UDOT Signals: $5500-5999$ 